\title{
Congenital Absence of Gall Bladder
}

\author{
SF KABIR ${ }^{\mathrm{a}}$, MS HAQUE ${ }^{\mathrm{b}}$
}

\begin{abstract}
Summary:
Congenital absence of gall bladder is a very rare entity found in clinical practices. Due to miss interpretation of sonographic findings as they are not familiar with the condition it possess a great difficulty in management the patient.
\end{abstract}

Though the patient present with the feature of acute cholecystitis, conservative treatment is enough to cure the

\section{Introduction}

Biliary system variants are relatively common but is isolated gallbladder agenesis is a rare entity with an estimated incidence of 10-65 per 100,000. Females are more commonly affected (ratio 3:1), typically presenting in the 2nd or 3rd decade of life. Despite an absent gallbladder, half of patients present with symptoms similar to biliary colic, which is poorly understood. Symptomatic congenital absence of gall bladder puts the diagnostic dilemma and treatment difficulties.

\section{Case Report:}

1. A 35 years old woman, mother of two children, of Noakhali District. Bangladesh, presented with severe upper abdominal pain, bouts of vomiting, slight abdominal distention. She was non-icteric, mildly anaemic.

Physical examination revealed good health, remarkable tenderness over the Right. hypochondriac region. Murphy's sign was positive. She was mildly dehydrated. Patient was normotensive and non diabetic. Examination of other systems revealed normal findings.

a. Dr. Sheikh Firoj Kabir, MCPS, FCPS (Surgery), Assoc. Prof. of Surgery, Tairunessa Memorial Medical College, Tongi, Dhaka, Bangladesh.

b. Dr. Md. Sirajul Haque, FCPS (Surgery), MS (Uro), Assoc. Prof. of Surgery, Comilla Medical College, Comilla, Bangladesh.

Address of correspondence: Dr. Sheikh Firoj Kabir, MCPS, FCPS (Surgery), Assoc. Prof. of Surgery, Tairunessa Memorial Medical College, Tongi, Dhaka, Bangladesh, E-mail: drfiroj@yahoo.com

Received: 14 May, 2011

Accepted: 11 June, 2012 disease. Modern radiological intervention make the diagnosis confirm, thus preventing unnecessary surgical procedure.

Here we report two cases of Congenital absence of gall bladder presenting as acute cholecystitis ultimately diagnosed and treated.

(J Bangladesh Coll Phys Surg 2012; 30: 177-180)

Routine laboratory examinations (Complete Blood Count, Erythrocyte sedimentation rate, blood biochemistry Panel) revealed nothing abnormal except Neutrophilic lucocytosis.

Liver function tests, serum Creatinine, Random blood sugar were within normal limit.

Ultrasonographic scan done but the comment was "gall bladder could not visualized may be due to bowel gas" (Fig.-1)

Contrast enhanced computed tomography (Fig.-2) revealed absence of gall bladder without any remarkable change in the extra and intra hepatic biliary apparatus.

Common bile duct was normal in diameter with no calculus present. Magnetic Resonance Cholangio Pancreatography done later (Fig.-3) which revealed absent gall bladder, normal intra and extra hepatic biliary channels. Common bile duct normal in diameter.

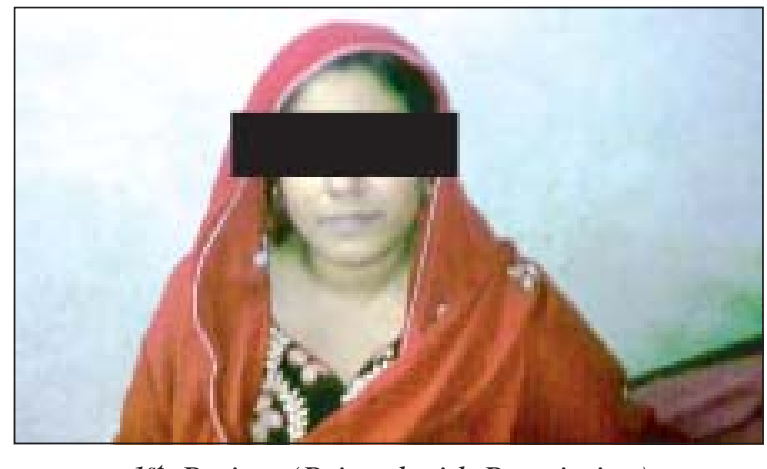

$1^{\text {st }}$ Patient (Printed with Permission) 


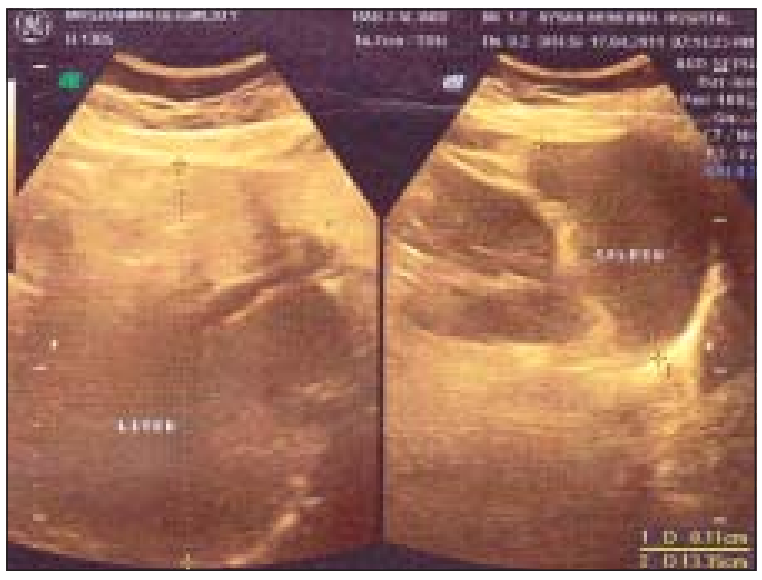

Fig.-1: Ultrasonographic Scan

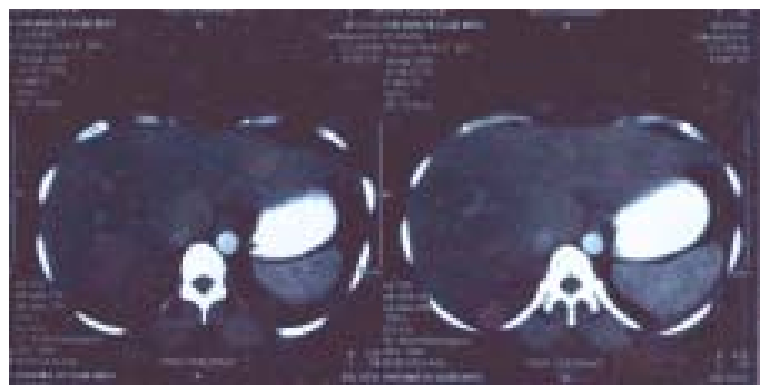

Fig.-2: CT Scan

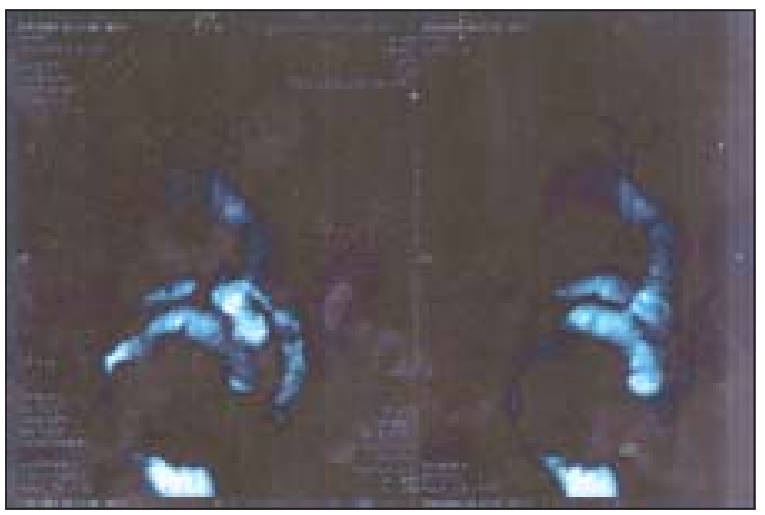

Fig.-3: MRCP Scan

Diagnostic laparoscopy done and gall bladder could not found. Common bile duct and Right and Left hepatic duct were found normal.

No other congenital abnormalities like annular pancreas or atresia found. The patient was treated conservatively with nothing per oral, intravenous fluid, Broad spectrum antibiotics, Nasogastic Suctions, Analgesics and antispasmodics.
2. The second patient 32 years old Bangladeshi woman, mother of two children, of Comilla District Bangladesh, admitted in a local clinic with severe upper abdominal pain, fever,nausea, and anorexia. Physical examination revealed good health, remarkable tenderness over the Right. hypochondriac region. Murphy's sign was positive. She was mildly dehydrated. Patient was hypertensive, non insulin dependent diabetic. Examination of other systems revealed normal.

Routine laboratory examinations revealed nothing abnormal except Neutrophilic lucocytosis.

Liver function tests, serum Creatinine were within normal limit.

Ultrasonographic scan done and the comment was "contracted gallbladder " (Fig.-4)

The patient underwent laparotomy but during the procedure gall blader was absent.The extra hepatic billiary apparatus were normal.

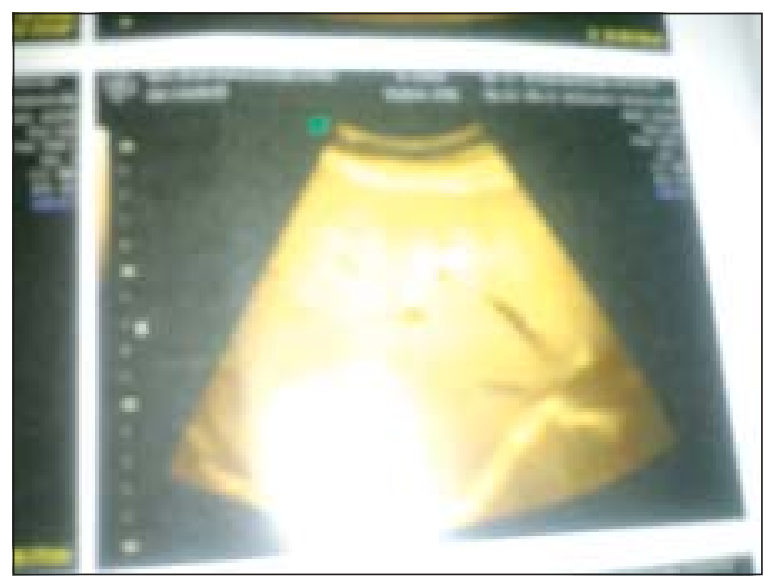

Fig.-4: Ultrasonographic Scan

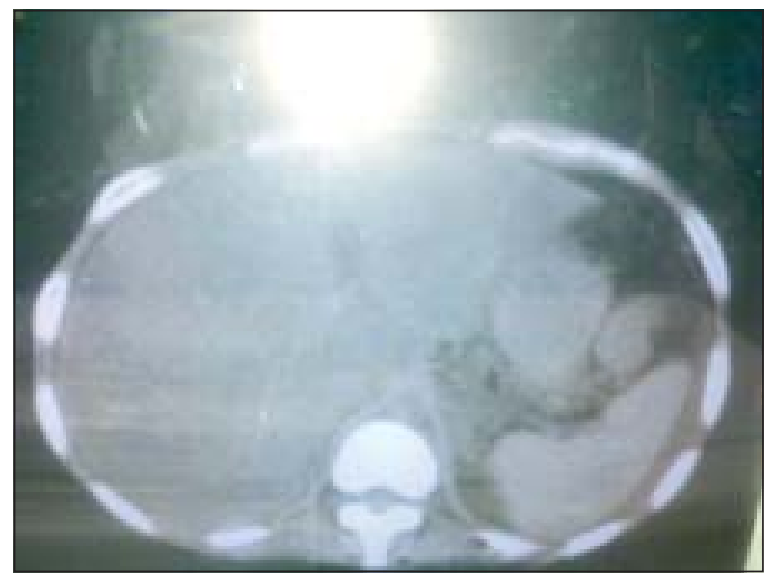

Fig.-5: CT Scan 


\section{Discussion:}

Gallbladder agenesis is a rare entity with an estimated incidence of $10-65$ per $100,000^{1,2}$. The incidence is noted to be higher (up to 90 per 100,000) in studies based on autopsy reports ${ }^{3}$. The first reports of cases of gallbladder agenesis date back to 1701 and 1702 by Lemery and Bergman ${ }^{1,2,4}$. The pathogenesis is related to embryonic development due to failure of the gallbladder and cystic duct to bud off from the common bile duct during the fifth week of gestation ${ }^{1}$.

Prior authors have classified patients into three groups. The first group consists of asymptomatic anatomical abnormalities seen incidentally on autopsy. The second group presents with symptoms of biliary colic (54\%), dyspepsia (34\%) and/or jaundice (27\%), and the third presents in childhood with other associated severe fetal anomalies ${ }^{2,4}$.

The exact prevalence of each of the three groups is variable based on published reports. It is thought that approximately $70 \%$ of cases are usually isolated anomalies, although some cases appear to be familial and are associated with more severe anomalies ${ }^{5,8,9}$. In an interesting series of 34 cases (29 children and 5 adults) of congenital gallbladder agenesis, the most common anomalies associated were involving the genitourinary tract followed by gastrointestinal and cardiovascular malformations. Family history was negative in all, suggesting a sporadic occurrence ${ }^{10}$.

Historically, all cases were identified intraoperatively. In a review of 9 cases by Cho et al. ${ }^{11}$, all patients underwent a laparotomy, which failed to identify the gallbladder. However, now with the increased frequency of advanced imaging, cases are being diagnosed more often and, more importantly, before any surgical intervention.

However, given that patients with gallbladder agenesis tend to present symptoms suggestive of biliary colic, a number of them are still diagnosed intraoperatively. Due to a lack of awareness of the diagnosis, this entity remains a diagnostic challenge ${ }^{2,12}$. In those cases which are diagnosed intraoperatively, patients often are exposed to complications from prolonged exploration ${ }^{13}$, and it is suggested to abort the procedure rather than complete further exploration if a gallbladder is not found on laparoscopy since open exploration for possible ectopic gallbladder increases the risk of complications ${ }^{14}$. Intraoperative ultrasound can demonstrate an ectopic gallbladder but is not always available ${ }^{13}$. A follow-up with more advanced imaging techniques should be the next option to truly identify gallbladder agenesis as the sole abnormality to guide management further.

It is therefore important to consider the presence of this unusual entity when the nonvisualization of the gallbladder is suggested on ultrasound ${ }^{15}$. However, as is known, ultrasound is highly dependent not only on the operator but also on other factors such as body habitus or presence of bowel gas obscuring visualization. Cases of gallbladder agenesis have been reported as 'contracted/fibrotic gallbladder' on ultrasound ${ }^{14}$.

HIDA scans, which are also usually performed in patients with cholecystitis, in this case are unhelpful since nonvisualization of the gallbladder remains typical of cystic duct obstruction, as well as of agenesis $^{13}$, MRCP is considered the test of choice if there is suspicion. It is also helpful in demonstrating an ectopic gallbladder along with other possible anomalies of the biliary tract system ${ }^{8}$ In terms of treatment, there are no specific guidelines on how to manage these cases. Interestingly, one author notes that $98 \%$ of patients had resolution of symptoms after exploratory, nontherapeutic surgery ${ }^{5}$. It is unclear how these patients would have had symptom resolution in the absence of exploration.

\section{Conclusions:}

Gallbladder agenesis presents as a significant diagnostic challenge. With the advances in imaging, more cases of gallbladder agenesis are being diagnosed incidentally and outside of the operating room. Clinicians should have a strong index of suspicion if nonvisualization is suggested by an ultrasound. A positive HIDA scan can be seen in the presence of gallbladder agenesis in the absence of cholecystitis. MRCP is considered the investigation of choice if there is suspicion. It is also helpful in demonstrating an ectopic gallbladder along with other possible anomalies of the biliary tract system. Management is usually conservative with smooth muscle relaxants. 


\section{References:}

1. Mittal A, Singla S, Singal R, Mehta V. Gallbladder agenesis with common bile duct stone: a rare case with a brief review of the literature. Turk J Gastroenterol. 2011;22:216-218. [PubMed]

2. Malde S. Gallbladder agenesis diagnosed intra-operatively: a case report. J Med Case Reports. 2010;4:285. [PMC free article] [PubMed]

3. Monroe SE, Ragen FJ. Congenital absence of the gallbladder. Calif Med. 1956;85:422-423. [PMC free article] [PubMed]

4. Bennion RS, Thompson JE, Tompkins RK. Agenesis of gallbladder without extrahepatic biliary atresia. Arch Surg. 1988;123:1257-1260. [PubMed]

5. Stephenson JA, Norwood M, Al-Leswas D, Al-Taan O, Beable R, Lloyd DM, Dennison AR. Hepatic haemangioma masquerading as the gallbladder in a case of gallbladder agenesis: a case report and literature review. HPB Surg. 2010 2010. pii: 971609.

6. Hershman MJ, Southern SJ, Rosin RD. Gallbladder agenesis diagnosed at laparoscopy. J R Soc Med. 1992;85:702-703. [PMC free article] [PubMed]

7. Wright E, Madore P. Congenital absence of the gallbladder. Can Med Assoc J. 1965;93:123-125. [PMC free article] [PubMed]
8. Fiaschetti V, Calabrese G, Viarani S, Bazzocchi G, Simonetti G. Gallbladder agenesis and cystic duct absence in an adult patient diagnosed by magnetic resonance cholangiography: report of a case and review of the literature. Case Report Med. 2009;2009:674-768.

9. Demir MK, Kilicoglu G. Rare coincidence of congenital short and annular pancreas with gallbladder agenesis and splenic malrotation. Br J Radiol. 2008;81:e204-e206. [PubMed]

10. Turkel SB, Swanson V, Chandrasoma P. Malformations associated with congenital absence of the gall bladder. J Med Genet. 1983;20:445-449. [PMC free article] [PubMed]

11. Cho CH, Suh KW, Min JS, Kim CK. Congenital absence of gallbladder. Yonsei Med J. 1992;33:364-367. [PubMed]

12. Gupta N, Gupta SK, Kapoor HS. Gallbladder and cystic duct agenesis diagnosed laparoscopically. Hepatobiliary Pancreat Dis Int. 2010;9:107-108. [PubMed]

13. Peloponissios N, Gillet M, Cavin R, Halkic N. Agenesis of the gallbladder: a dangerously misdiagnosed malformation. World J Gastroenterol. 2005;11:6228-6231. [PubMed]

14. Balakrishnan S, Singhal T, Grandy-Smith S, El-Hasani S. Agenesis of the gallbladder: lessons to learn. JSLS. 2006;10:517-519. [PMC free article] [PubMed]

15. Kabiri H, Domingo OH, Tzarnas CD. Agenesis of the gallbladder. Curr Surg. 2006;63:104-106. [PubMed] 